\title{
Transient analysis of systems exhibiting inverse response and their control with CSTR as a case study
}

\author{
Rohan Lakhani ${ }^{1}$, C. R. Srinivasan ${ }^{2}$, Srividya $\mathbf{R}^{3}$ \\ ${ }^{1,2}$ Department of Instrumentation and Control Engineering, Manipal Institute of Technology, \\ Manipal Academy of Higher Education, India \\ ${ }^{3}$ Department of Eletrical and Eletronics Engineering, Manipal Institute of Technology, \\ Manipal Academy of Higher Education, India.
}

\begin{tabular}{|c|c|}
\hline Article Info & ABSTRACT \\
\hline Article history: & In this paper the phenomenon of inverse response from systems was \\
\hline Received Dec 26, 2019 & $\begin{array}{l}\text { investigated and its transient response thoroughly analyzed. Inverse response } \\
\text { is shown by non-minimum phase systems and some minimum phase systems. }\end{array}$ \\
\hline Revised Mar 28, 2020 & Transient analysis of these systems is lacking in literature A case study was \\
\hline Accepted Apr 11, 2020 & done for a non-linear, non-minimum phase CSTR (Continuous Stirred Tank \\
\hline Keywords: & $\begin{array}{l}\text { implemented on other systems as well, which exibhit inverse responses, } \\
\text { including model reference adaptive control. The finding shows that PID goes }\end{array}$ \\
\hline Adaptive control & with the inverse response, is unable to suppress it and requires large control \\
\hline Inverse response & area where PID is lacking with these systems. MRAC shemes were able to \\
\hline Model reference adaptive control & overcome all these issues. For CSTR also, these findings hold true thus \\
\hline Non-minimum phase & points at using advance stratigies in process control industries for maximzing \\
\hline Optimization & product yield. \\
\hline
\end{tabular}

Copyright $@ 2020$ Institute of Advanced Engineering and Science. All rights reserved.

\section{Corresponding Author:}

C R Srinivasan,

Department of Instrumentation and Control Engineering,

Manipal Institute of Technology, Manipal Academy of Higher Education,

Manipal, Karnataka, India.

Email: cr.srinivasan @manipal.edu

\section{INTRODUCTION}

The inverse response is shown by all non-minimum phase systems, if an input is applied then the system first moves in the opposite direction before moving in the right direction. Dc-Dc boost converter, hydro turbine of hydroelectric power plants, coupled tank system [1] are some of the many non-minimum phase systems showing inverse responses. An interesting phenomenon which is found in [2] is that right hand sides zeros are not necessary for system to show inverse response. Adaptive control of aircraft with nonminimum phase zeros was performed in [3], robust control for non-minimum phase boiler [4] with LQG control, design of controller for XY non-minimum phase table was performed [5], U-model design for boieng 747 altitude control [6]. As it can be seen in literature previous works are done on specific non-minimum phase plants, inverse systems are only associated with non-minimum systems which is not true as shown in [2]. The transient analysis of inverse systems is lacking in literature, which can be very crucial. In control literature the standard non-minimum phase systems which are generally dealt assume that the system is composed of two first order systems, one of them is faster than the other and the slower process is the one which reaches its steady state value sooner, this gives rise to an inverse response. But this is not the case in most processes, system can be of higher order, have complex poles and more than one right hand side zero.

CSTR is a benchmark process in industries and shows inverse response, generally arbitrary temperature feed is given to the system which yields less efficent performance in terms of time taken for 
obtaining final products in chemical reactions. Its non-linearity is also a challenge, controllers were developed in [7-11] including Sliding model [9], model predictive control [11] for CSTR, but tracking was hindered by the initial inverse response. [7-11, 12] arbitrary temperature trajectories were used, PID still plays an important role in process industries but its use for these systems is not looked at carefully. Thus, there is a need to for developing an optimized trajectory for input temperature and control schemes which are able to suppress inverse response while showing good tracking bhevaiour. In addition to the CSTR case study three other systems were also looked at and performances evaluated.

A coupled tank system which is a non-minimum phase standard plant, another non-minimum phase transfer function having complex poles and higher order system which has all of its zeros in the left half plane and still shows inverse response. Three control schemes are compared for the coupled tank system namely PI inner-outer factorization controller, Feedthrough compensator, quantitative controller. For system with complex poles and higher order system, adaptive controllers namely Adaptive PID, 1-Dof MRAC and 2-Dof MRAC are used.

Quantifying the inverse response-

Figure 1(a) shows the inverse responses which has an initial undershoot. Figure 1(b) crosses zero more than once, this behavior can be predicted -

Theorem1: If G(s) has $n_{+}$positive zeros in the ROC and zero at $s=0$ of multiplicity $n_{o}\left(n_{+}, n_{o} \in\right.$ $W)$, then output has at least $\left(n_{+}+n_{o}-1\right)$ or $n_{+}$number of zero crossings. [1]

Theorem2: The system has a relative degree 1 or has at least one real pole closest to the origin, having odd number of positive zeros [1]. The magnitude of the initial undershoot can be given as $M \propto$ $e^{-\left(\left|z_{c}\right|-\left|p_{c}\right|\right)}$, if the system satisfies Theorem 2 . Where $z_{c}$ and $p_{c}$ are the real part of complex zero and real pole closest to the origin respectively.

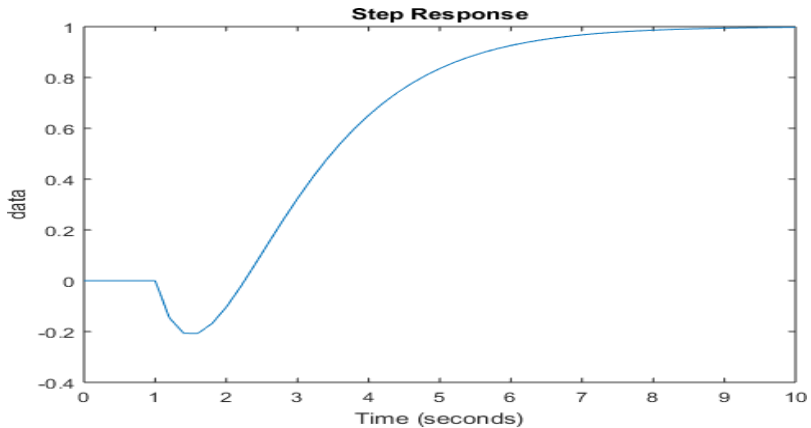

(a)

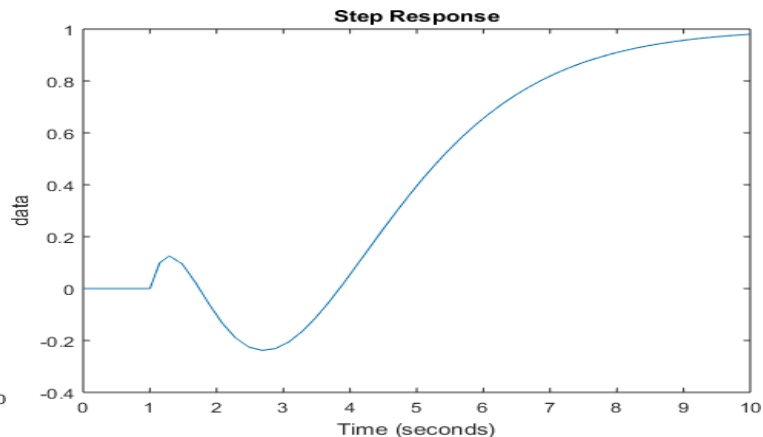

(b)

Figure 1. Inverse response of non-minimum phase system (a) one zero crossing with initial undershoot (b) multiple zero crossings

\section{RESEARCH METHOD}

2.1. Controller design

\subsubsection{Controllers specifically for non-minimum phase systems}

These controllers are for standard non-minimum phase systems such as shown in Figure 2. The tuning of the controllers has been done by taking the coupled tank system as the process/plant.

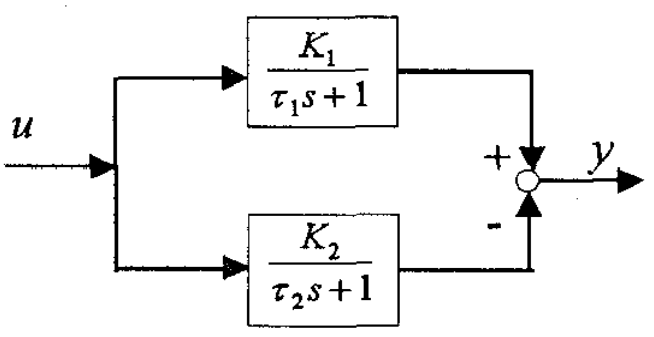

Figure 2. Standard non-minimum phase plant 
a) Quantitative Controller is given by-,

It is an extended version of smith's predicator based on $H_{\infty}$ control [13]. The controller after tuning

$$
C(s)=\frac{\left(25.08 s^{2}+23.9 s+1\right)}{\left(0.40552 s^{2}+1.50552 s+1\right)}
$$

The plant is decomposed into two first order systems which are used in the feedback path. The compensator scheme is shown in Figure 4(a).

b) PI inner-outer factorization

This scheme is implemented to remove the non-minimum phase dynamics from the control loop [14]. It has a PI controller and two feedback compensators given by-

$$
\begin{aligned}
& C(s)=14.18+\frac{3.672}{s} \\
& N(s)=\frac{(-1.1 s+1)}{(1.1 s+1)} \\
& M^{-1}(s)=\frac{1}{1+22.8 s}
\end{aligned}
$$

Where $C(s)$ is a pi controller, $N(s)$ and $M^{-1}(s)$ are part of the feedback compensation. The scheme can be seen in the Figure 4(b).

c) Feedthrough compensator

In this a compensator is chosen such that the closed loop system becomes minimum phase and with high gain the system is stabilized. The compensator chosen is $T(s)=1 /\left(s^{2}+5 s+2\right)$ and the gain $K=74$. The scheme [15] is shown in Figure 5(a).

\subsubsection{Controllers for non-minimum phase system with complex poles and minimum phase higher} order system showing inverse response

a) Adaptive PID

The approximate version of the adaptive PID is implemented which does not require any plant knowledge [16]. The adaptive law is given as-,

$$
\begin{aligned}
& \dot{K}_{P}=-\gamma e y_{1} \\
& \dot{K}_{I}=-\gamma e y_{2} \\
& \dot{K}_{D}=-\gamma e y_{3}
\end{aligned}
$$

$\gamma$ is the adaptive gain, $\mathrm{e}$ is the error signal and $y_{1}, y_{2}, y_{3}$ are outputs from first three subsystems. The scheme is shown in Figure 5(b).

b) Dof model reference adaptive control

The adaptive law is given as-,

$$
\begin{aligned}
& \dot{K}_{c}=-\gamma e y_{m}(t) \\
& e=y_{p}-y_{m}
\end{aligned}
$$

where $\gamma$ the adaptive gain, $y_{m}(t)$ is the output from the reference model and $e$ is the error between the plant and reference model output [17]. The controller is shown in Figure 6(a).

c) Dof model reference adaptive control

This a 2-Dof implementation of MRAC [18],

$$
\dot{\theta_{1}}=-\gamma_{1} y_{p} G_{m} e
$$




$$
\dot{\theta_{2}}=-\gamma_{2} r G_{m} e
$$

Where $\gamma_{1}, \gamma_{2}$ are the adaptive gains. $y_{p}, r, e, G_{m}$ are the plant output, reference input, error and reference model respectively. The scheme can be seen in Figure 6(b).

The adaptive law is given by-,

$$
K_{c}=r \theta_{1}-y_{p} \theta_{2}(12)
$$

\subsection{System Identification of non-linear, non-minimum phase CSTR}

The input signal used for the identification of real time CSTR is APRBS, as shown in Figure 3 and a transfer function was approximated using system identification toolbox in matlab.

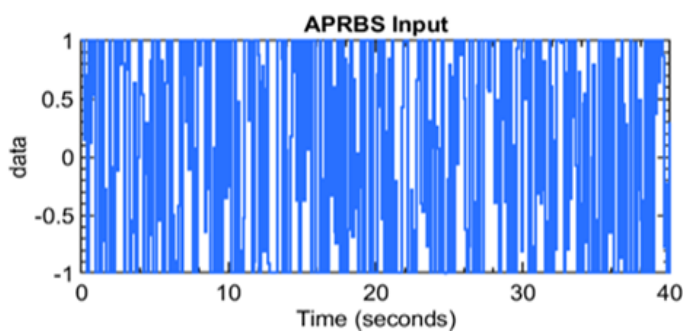

(a)

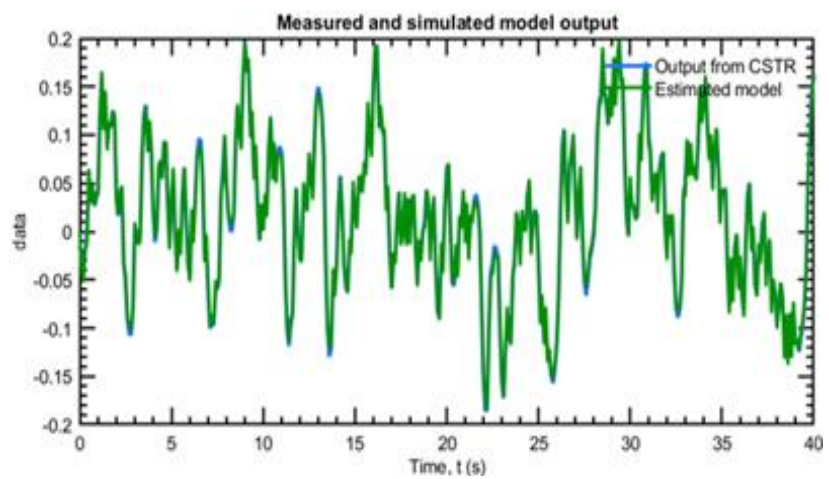

(b)

Figure 3. (a) Input APRBS signal to CSTR (b) Output measured and estimated output

The estimated transfer function is given by,

$$
G p(s)=\frac{0.4(-0.4 s+1)}{(0.6 s+1)(0.5 s+1)}
$$

\subsection{Novel optimized temperature trajectory for CSTR using acado}

An optimized trajectory is important in industries as by using this the final product in any chemical reaction can be maximized in a fixed time interval which increases efficieny as well as profits. The chemical reaction and model parameters for the differential equations for the reactor, which can be used for this CSTR system as well are given in [19].

The differential equation used for the optimization is given by-

$$
\begin{aligned}
& \frac{d C_{A}}{d t}=-k o e^{-\frac{E R}{T}} C_{A}{ }^{2} \\
& \frac{d T}{d t}=-\frac{H}{M c p} e^{-\frac{E R}{T}} C_{A}{ }^{2}-\frac{U A}{M c p}(T c-T)
\end{aligned}
$$

This becomes a non-linear constrained dynamic optimization problem and the constraints are initial temperature, bounds in jacket temperature. The jacket temperature which is the manipulated variable in this case. But for code generation is taken as control varibable, having a different context in optimization. An overview of acado and optimization can be found in [20] which is left to the interested reader.

$$
\begin{aligned}
& \underset{u}{\max } J=-g(x, u) \\
& \dot{X}=f(x) \\
& 20^{\circ} \mathrm{C}<\text { Tjacket }<50^{\circ} \mathrm{C} \\
& T=25^{\circ} \mathrm{C}
\end{aligned}
$$


In (16)-(19) represents the lagrange cost function, which is to minimize the reactant $\mathrm{A}$ in a fixed time interval with constraints on initial temperature and allowable jacket temperature. The acado matlab code is given as,

\section{BEGIN_ACADO;}

acadoSet('problemname', 'CSTR');

DifferentialState ca $\mathrm{T} \%$ defining differential states

Control $\mathrm{Tj} \%$ control variable

$\mathrm{c} 2=0.02881$;

$\mathrm{c} 1=-5.79 ; \%$ constants

$\mathrm{E}=13550$

$\mathrm{ko}=4.7 * 10^{\wedge} 19$;

$\mathrm{f}=$ acado.DifferentialEquation () ;

f.add $(\operatorname{dot}(\mathrm{ca})==-\mathrm{ko} * \exp (-\mathrm{E} / \mathrm{T}) * \mathrm{ca} * \mathrm{ca}) ; \%$ differential equations

f. $\operatorname{add}(\operatorname{dot}(\mathrm{T})==-\mathrm{c} 1 * \mathrm{ko} * \exp (-\mathrm{E} / \mathrm{T}) * \mathrm{ca} * \mathrm{ca}-\mathrm{c} 2 *(\mathrm{~T}-\mathrm{Tj}))$;

ocp $=$ acado. $\operatorname{OCP}(0,100,100) ; \%$ number of steps and final time

ocp.minimizeLagrangeTerm(ca); \% minimizing the Lagrange term, final concentration of reactant A ocp.subjectTo( f);

ocp.subjectTo( 'AT_START', ca ==0.9); \% initial concentration of A

ocp.subjectTo( 'AT_START', T == 298.16); \% initial temperature

ocp.subjectTo( $293.15<=\mathrm{Tj}<=323.15)$; \% jacket temperature constraint

algo = acado.OptimizationAlgorithm(ocp);

algo.set('INTEGRATOR_TOLERANCE', 1e-1 ); \% tolerance for convergence

-END_ACADO;

out $=$ CSTR_RUN ()$; \%$ run command

plot(out.STATES(:,1), out.STATES(:,3))

\subsection{Control algorithm}

All control schemes are implemented using Matlab Simulink environment. The following shows the control schemes with their respective plant transfer functions,

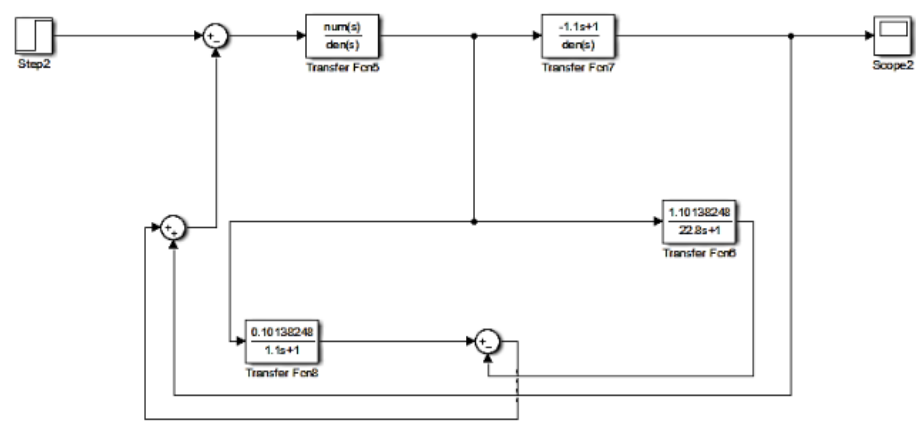

(a)

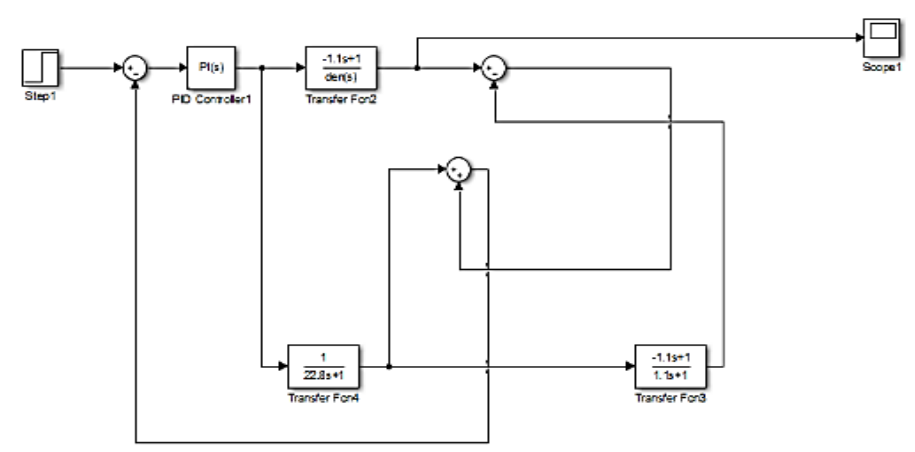

(b)

Figure 4. (a) Quantitative controller (b) PI inner-outer factorization 


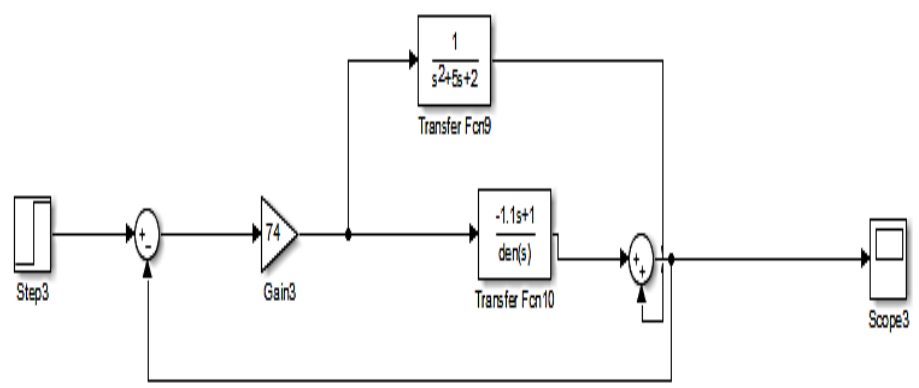

(a)

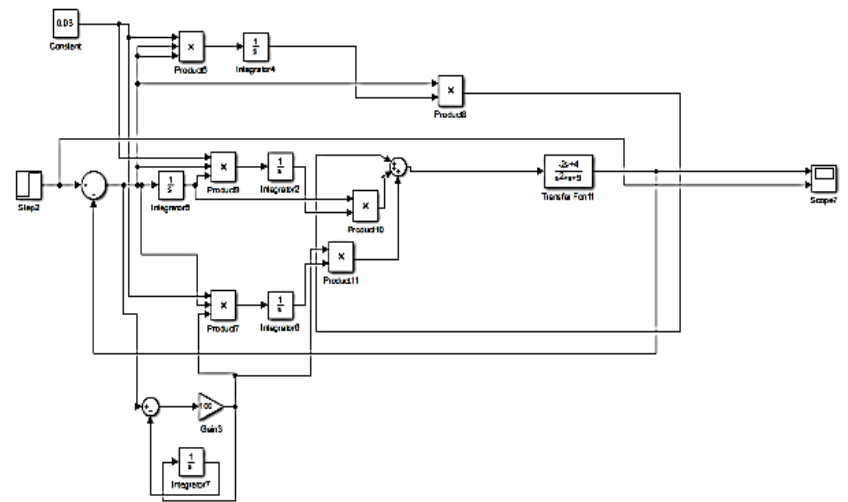

(b)

Figure 5. (a) Feedthrough compensator (b) Adaptive PID

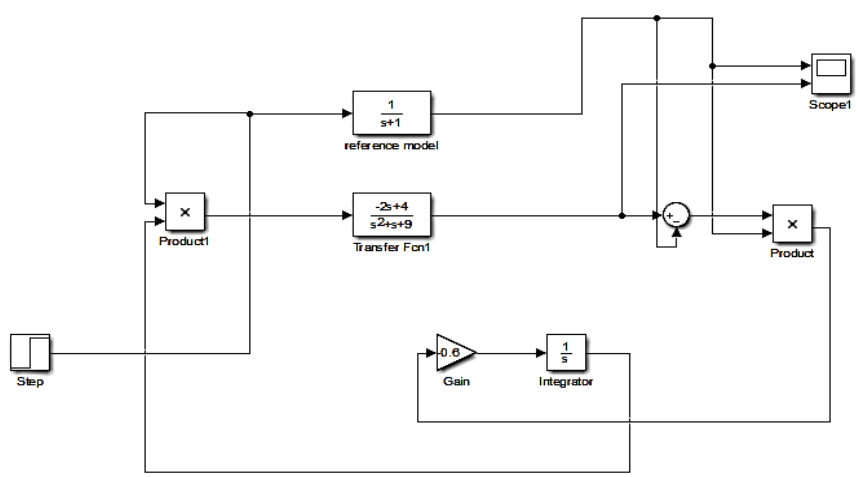

(a)

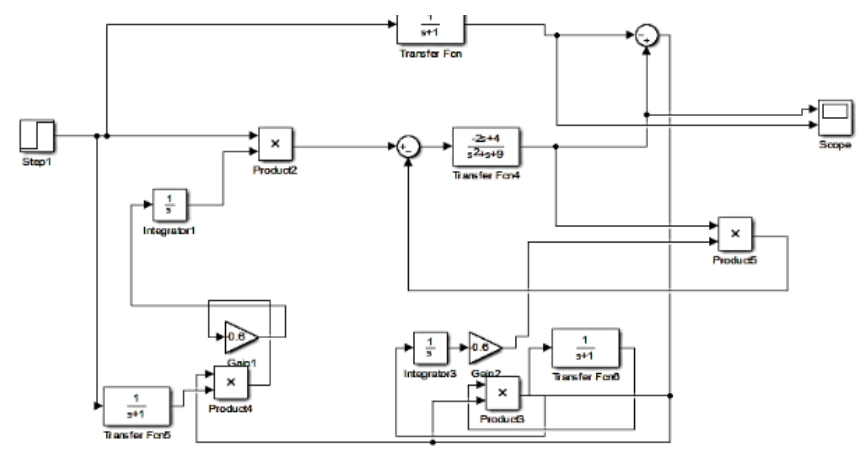

(b)

Figure 6. (a) 1-Dof MRAC (b) 2-Dof MRAC 


\section{RESULTS AND DISCUSSION}

\subsection{Standard non-minimum phase system}

The coupled tank system $G_{1}(s)[1]$ which is a non-minimum phase system with real roots is considered. The Transfer function is given as-,

$$
G_{1}(s)=\frac{(1-1.1 s)}{(1+1.1 s)(1+22.8 s)}
$$

The controllers were tuned using the plant parameters and the PID was tuned using Ziegler-Nichols, the PID parameters are $\mathrm{P}=14.18, \mathrm{I}=3.672, \mathrm{D}=13.69$.

\subsubsection{Step response}

It can be seen in Figure 7, that the feedthrough compensator gives the least initial undershoot, but due to its oscillatory behavior and overshoot, PI input-output factorization controller is chosen for this plant as a compromise is achieved between initial undershoot and system performance. PID shows a large initial undershoot in Figure 7, the performances are compared in Tables 1 and 2.

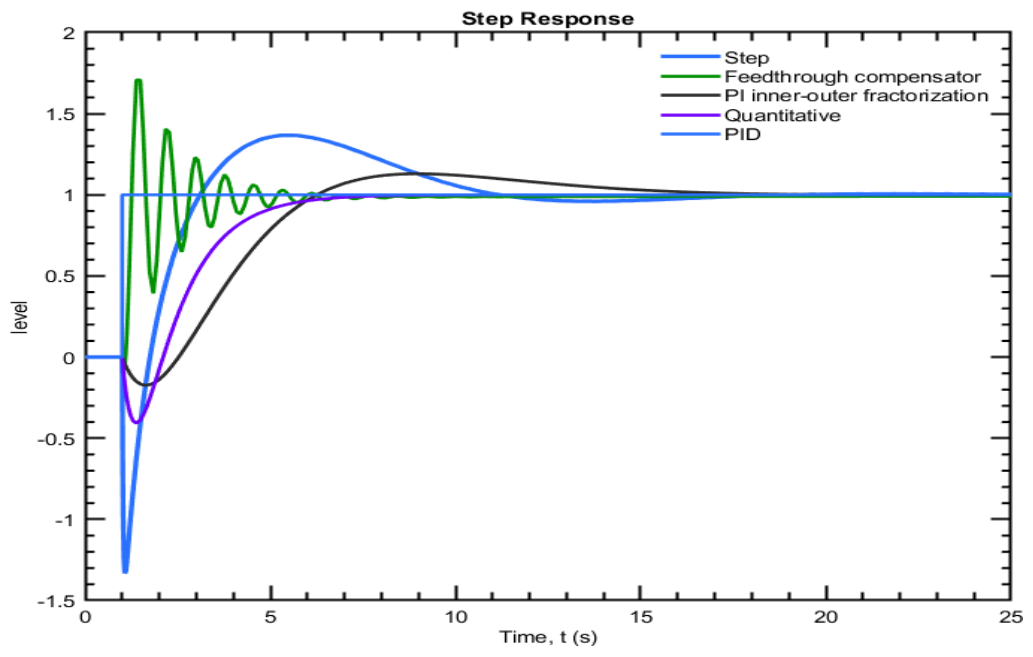

Figure 7. Step response of all control schemes with PID-ZN as a benchmark

Table 1. Comparison of system performance index with different control schemes

\begin{tabular}{ccccc}
\hline & PID & Feedthrough compensator & Quantitative Controller & PI inner-outer factorization \\
\hline Settling time $(\mathrm{sec})$ & 16.2 & 11.6 & 6.8 & 15.8 \\
Rise time $(\mathrm{sec})$ & 2.85 & 1.24 & 4.8 & 5.6 \\
Overshoot $(\%)$ & 36 & 77 & 0 & 13 \\
\hline
\end{tabular}

Table 2. Comparison of inverse response form different controllers

\begin{tabular}{ccc}
\hline & Initial undershoot & Number of zero crossing \\
\hline PID & -1.34 & 1 \\
Feedthrough Compensator & -0.075 & 1 \\
Quantitative Controller & -0.4 & 1 \\
PI inner-outer factorization & -0.174 & 1 \\
\hline
\end{tabular}

\subsubsection{Robustness analysis}

Figure 8 shows response of the system to input disturbances for a train of pulses, PID gives the maximum deviation of 0.25 and the other controllers have negligible deviation. The poor robustness of PID can be explained due to the systems low gain margin, a problem with non-minimum phase system [21]. As the closed loop gain increases system poles move towards the righthand side zeros. 


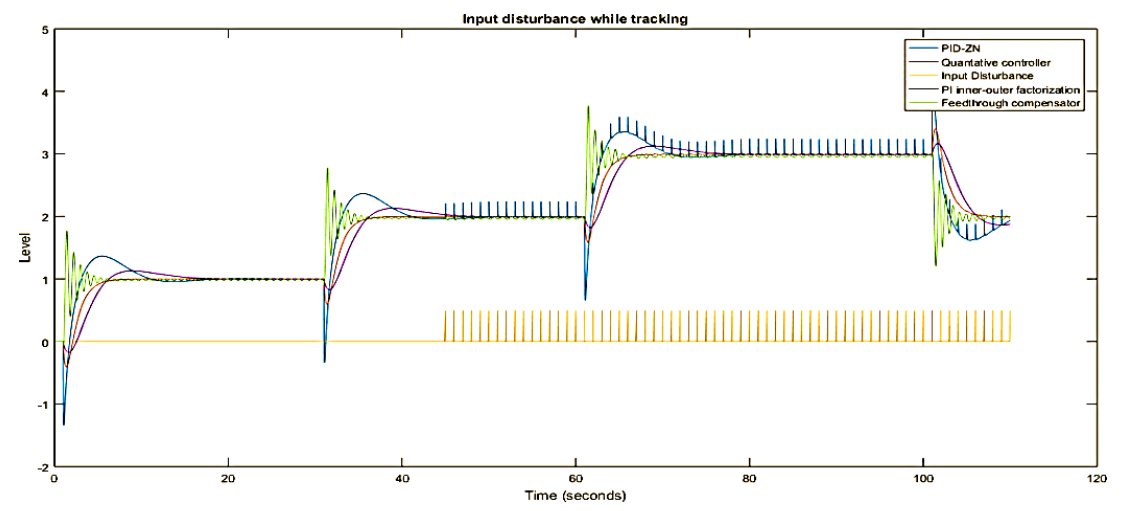

Figure 8. Input disturbance response while tracking

\subsection{Non-minimum phase system with complex roots}

The controllers implemented before, work on the assumption that the system is a standard system having two real poles, which is popular in control literature.

But system can be of higher order with complex poles thus adaptive schemes have been used for controlling this system. The system $G_{2}(s)$ is given as in [22] having complex roots-,

$$
G_{2}(s)=\frac{(-2 s+4)}{\left(s^{2}+s+9\right)}
$$

The PID-ZN tuning rules for this system were $\mathrm{P}=0.6749, \mathrm{I}=0.539, \mathrm{D}=0.2113$.

\subsubsection{Step response}

Adaptive controller parameters i.e. adaptive gains are given in Table 3, the reference model chosen for 1-Dof and 2-Dof MRAC is $1 /(s+1)$.

Table 3. Adaptation gain values of controllers

\begin{tabular}{ccc}
\hline & $\boldsymbol{\gamma}_{\mathbf{1}}$ & $\boldsymbol{\gamma}_{\mathbf{2}}$ \\
\hline Adaptive PID & 0.03 & NA \\
1-Dof MRAC & 0.6 & NA \\
2-Dof MRAC & 0.62 & 0.62 \\
\hline
\end{tabular}

Step response is shown in Figure 9 and performances are compared in Table 4 and Table 5, it can be seen that there are multiple zero crossings for PID and Adaptive PID controller. PID also gives the largest initial undershoot. The best performance is obtained by 2-Dof MRAC controller.

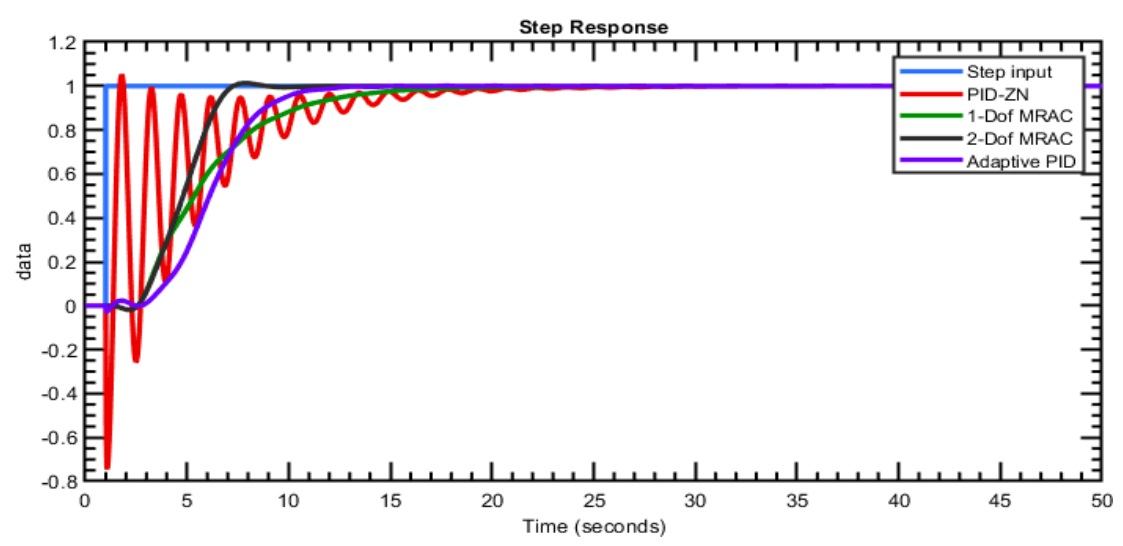

Figure 9. Step response of all adaptive control schemes with PID-ZN as a benchmark 
Table 4. Comparison of inverse response form different controllers

\begin{tabular}{ccc}
\hline & Maximum initial undershoot & Number of zero crossing \\
\hline PID & -0.75 & 3 \\
1-Dof MRAC & -0.02 & 1 \\
Adaptive PID & -0.028 & 3 \\
2-Dof MRAC & -0.02 & 1 \\
\hline
\end{tabular}

Table 5. Comparison of system performance index with different control schemes

\begin{tabular}{ccccc}
\hline & PID & 1-Dof MRAC & 2-Dof MRAC & Adaptive PID \\
\hline Settling time(sec) & 35 & 15.4 & 7 & 11 \\
Rise time(sec) & 1.64 & 10.5 & 6.4 & 8.8 \\
Overshoot(\%) & 5 & 0 & 1 & 0 \\
\hline
\end{tabular}

\subsubsection{Controller effort}

The controller effort is shown in Figure 10, conventional PID requires high control effort which can be a serious limitation in hardware. The initial high control output of PID can be attributed for trying to suppress the system inverse response.

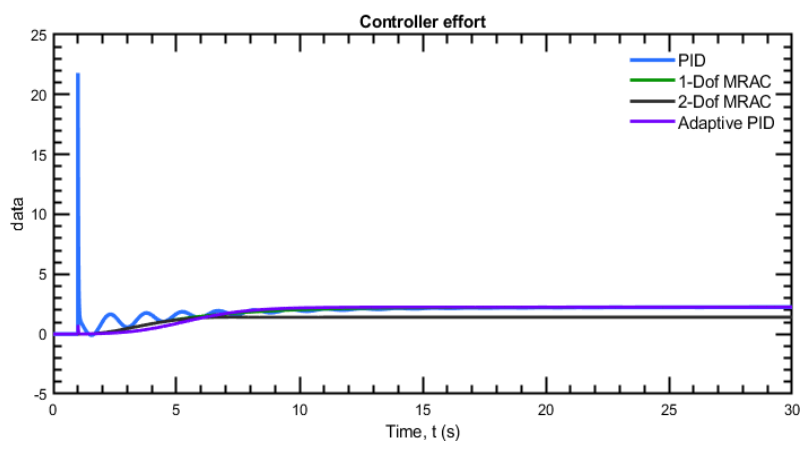

Figure 10. Control effort

\subsubsection{Higher order system with zeros on the left half plane}

In Figure 11(a) it can be seen that the system $G_{3}(s)$ shows an inverse response even though it has all zeros on the left half plane. For an inverse response the system does not necessarily have to be non-minimum phase. The system transfer function $G_{3}(s)$ is given as in [2]. Performance of the adaptive controllers is shown in Figure 11(b), all adaptive controllers were able to eliminate the inverse response and have zero initial undershoots.1-Dof MRAC gives the optimal performance.

$$
G_{3}(s)=\frac{\left(7.688 s^{5}+6.043 s^{4}+9.882 s^{3}+5.594 s^{2}+2.088 s+0.7047\right)}{\left(0.1896 s^{6}+2.078 s^{5}+9.12 s^{4}+20.07 s^{3}+22.21 s^{2}+10.17 s+0.2639\right)}
$$

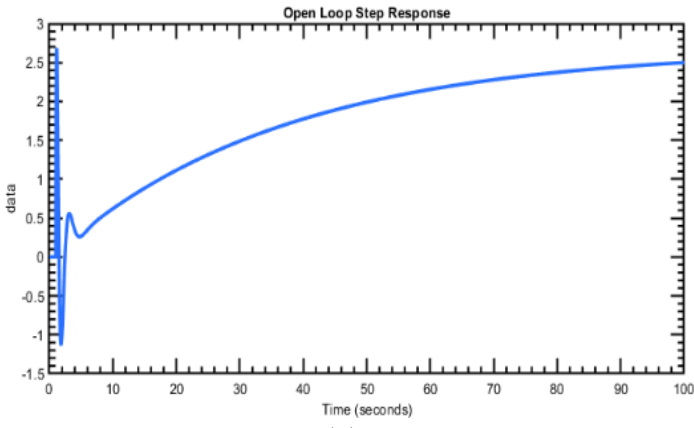

(a)

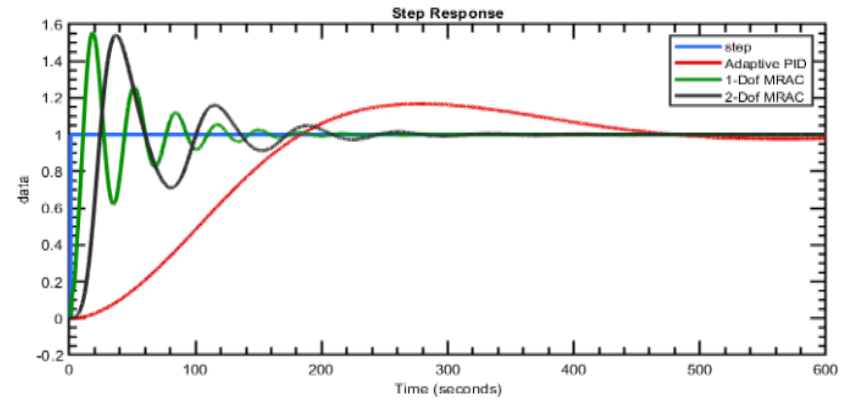

(b)

Figure 11. (a) Open loop step response of higher order system (b) Step response of higher order system with adaptive controller 


\subsection{A continious stired tank reactor case study, with novel optimized trajectory for temperature}

Here a non-minimum phase CSTR system is identified and the optimized trajectory is used for reference tracking. The significace of an optimium trajectory can be seen in [23], thus it becomes interesting to see how a popular system in process industries showing inverse response behaves with an optimum feed, shown in Figure 12 as it has impications in terms of the time, profits and product quality. A desired trajectory was used in [24] and an optimized trajectory was formed in [25].

The values of temperature in the figure has been scaled down for representation. The performance can be seen in Figure 13, PID is unable to suppress the inverse response and tracks the reference with oscillations but other advanced schemes are giving a good response. Figure 14 and 15 shows the conversion profile of reactant $\mathrm{A}$ to product $\mathrm{B}$, it can be seen that when optimum feed is used the conversion is much faster when compared to the feed which uses step input. The results were obtained for Figures 14 and 15 using ode45 solver in matlab.

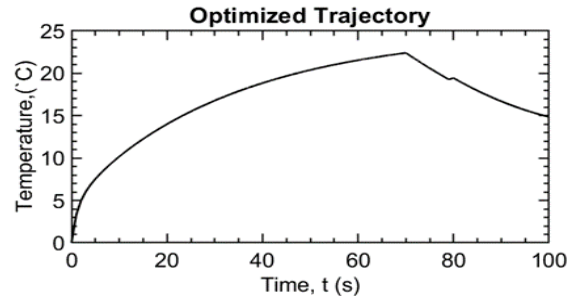

Figure 12. Optimized Trajectory for nonminimum phase, non-linear CSTR

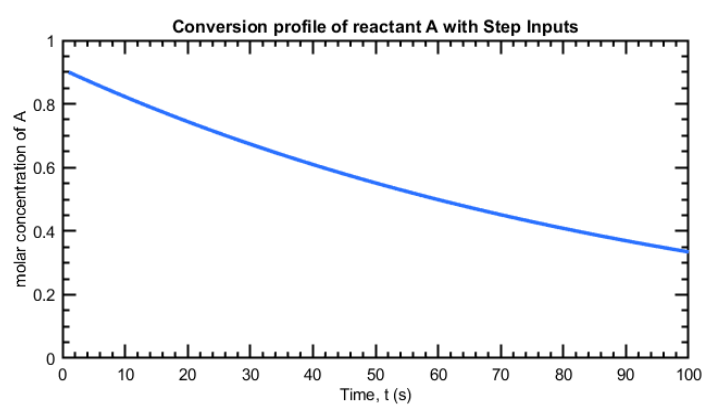

Figure 14. Conversion of A during the reaction with step input

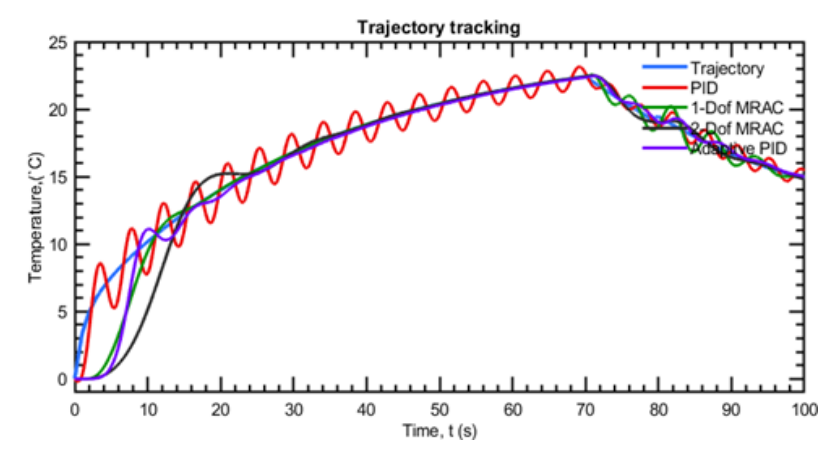

Figure 13. CSTR performance with input oprimized trajectory for tracking with different control schemes

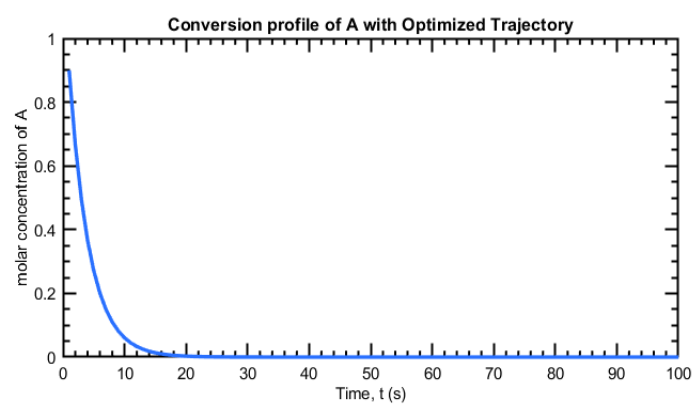

Figure 15. Conversion of A during the reaction with optimized trajectory

\section{CONCLUSION}

Analysis of three systems were done and performance compared, PI inner-outer factorization controller gives the best performance in terms of minimum initial undershoot and tracking performance for standard non-minimum phase coupled tank system. In adaptive control, 2-Dof MRAC gives superior performance for general non-minimum phase systems.

The novel optimum trajectory for CSTR was formed by solving a non-linear dynamic constrained optimization problem using acado and it was shown that, for a particular chemical reaction the reactant is converted to the product in a much shorter time, adaptive control was used for CSTR which suppressed the inverse response and gave good tracking performance for the trajectory. PID which is the workhorse in process industries has limitations when systems exhibiting inverse response is concerned as shown for CSTR. Robustness, high control effort and large initial undershoot are problems found with the conventional controllers and other previous schemes in laterature which were overcome using Adaptive control schemes. Tracking was not hindered by using MRAC techniques, inverse response was suppressed, maximum product was achieved in minimum amount of time with low control effort. 


\section{REFERENCES}

[1] S. Bose, Y. V. Hote, S. D. Hanwate, "Analysis of practical non-minimum phase systems by transfer function approach", 4th International Conference on Electrical Energy Systems (ICEES 2018), pp. 1-6, 2018.

[2] J.M. Maciejowski, "Right-half plane zeros are not necessary for inverse response", 2018 European Control Conference (ECC), 2018.

[3] Kelley E. Hashemi, Nhan T. Nguyeny, "Adaptive Maneuver Load Alleviation for Flexible Wing Aircraft with Nonmiminum Phase Zeros", 2018 AIAA Guidance, Navigation, and Control Conference, 2018.

[4] H. Zargarzadeh, M.R. Jahed Motlagh, "Robust Optimal Controller Design for a Non-minimum Phase Boiler System with a saturable Actuator", IEEE Multi-conference on Systems and Control Saint Petersburg, 2009.

[5] Michael Jackson Patrick et al., "Contouring control of non-minimum phase XY table system using trajectory ZPETC", International Journal of Scientific \& Technology Research, vol. 1, no. 6, pp. 80-85, 2012.

[6] Ji Qiu et al., "A U-model based controller design for non-minimum phase systems: Application to Boeing 747 Altitude-hold Autopilot", The 9th International Conference on Modelling, Identification and Control (ICMIC 2017), Kunming, China, July 10-12, 2017.

[7] Ruiyao Gao et al,“A nonlinear PID controller for CSTR using local model networks”, Proceedings of the 4th World Congress on Intelligent Control and Automation, China.

[8] Xi Lian, "Controller design of CSTR based on Kalman filter", Proceedings of the 30th Chinese Control Conference, China.

[9] Tabassum Rasul, Monisha Pathak, "Control of nonlinear chemical process using sliding mode control", IEEE 1st International Conference on Power Electronics, Intelligent Control and Energy Systems (ICPEICES), India.

[10] De-Xin Gao, Huan Liu, "Optimal dynamic control for CSTR nonlinear system based on feedback linearization", The 27th Chinese Control and Decision Conference, 2015.

[11] JaroslavPekař, VladimírHavlena, "Control of CSTR using model predictive controller based on mixture distribution", 6th IFAC Symposium on Nonlinear Control Systems, Germany.

[12] J. Mason C, Kambhampati, "Predictive control of a mixing tank using radial basis function networks", Proceedings of 35th IEEE Conference on Decision and Control.

[13] Zhang Wei-Dong and Sun You-Xian, "Quantitative Control of Inverse Response process," in Proc. of American Control Conference, Albuquerque, New Mexico, pp.3257-3261, 1997.

[14] S. Alcántara, C. Pedret, R. Vilanova, P. Balaguer, and A. Ibeas. "Control configuration for inverse response processes." Proc. of the 16th Mediterranean Conference on Control and Automation (MED08), 2008.

[15] Misra, Pradeep; "On the Control of Non-Minimum Phase Systems," American Control Conference, 1989, pp. 1295-1296,21-23 June 1989.

[16] B. M. Badreddine and F. Lin, "Adaptive PID Controller for Stable Unstable Linear and Non-Linear Systems," presented at the 2001 IEEE International Conference on Control Applications, Mexico City, 2001.

[17] P. Jain and M. J. Nigam, "Design of a Model Reference Adaptive Controller Using Modified MIT Rule for a Second Order System," Advance in Electronic and Electric Engineering, vol. 3, pp. 477- 484, 2013.

[18] M. Pal, G. Sarkar, R. K. Barai, T. Roy, "Design of Adaptive Two-Degree-of-Freedom Controller for Inversion based Non-Minimum Phase System", Proceedings of Michel Faraday IET International Summit-2015 (MFIIS2015), vol. 1, pp. 78-83, 12-13 September, 2015.

[19] Eduardo J. Adam, "Nonlinear batch reactor temperature control based on adaptive feedback based ilc", International Journal of Instrumentation and Control Systems, vol. 5, no. 1.

[20] B. Houska, H. Ferreau, M. Diehl, "ACADO toolkit-an open-source framework for automatic control and dynamic Opt.", Optim. Control Appl. Methods, vol. 32, no. 3, pp. 298-312, Jun. 2011.

[21] B. Hoagg, D.S. Bernstein, "Nonminimum-phase zeros - much to do about nothing - classical control - revisited part II", IEEE Control Systems Mag., vol. 27, no. 3, pp. 45-57, 2007.

[22] G. F. Franklin, J. D. Powell, A. Emami-Naeini, Feedback Control of Dynamic Systems, New Jersey: Prentice Hall, 2002.

[23] Bonnard B., Chyba M. "Singular Trajectories in Optimal Control", Baillieul J., Samad T. (eds) Encyclopedia of Systems and Control. Springer, London.

[24] G. R. Cho, P. H. Chang, Y. Jin, "Enhanced feed forward control of non-minimum phase systems for tracking predefined trajectory", Industrial Electronics (ISIE) 2010 IEEE International Symposium, pp. 167-172, 4-7 July 2010.

[25] C. Ko, K. Young and Y. Hsieh, "Optimized trajectory planning for mobile robot in the presence of moving obstacles," 2015 IEEE International Conference on Mechatronics (ICM), Nagoya, 2015, pp. 70-75, 2015, doi: 10.1109/ICMECH.2015.7083950 